\title{
Fever of unknown origin in returning travellers
}

\author{
Krzysztof Korzeniewski ${ }^{1}$, Bartłomiej Gaweł ${ }^{2}$, Dagny Krankowska² ${ }^{2}$ Katarzyna Wasilczuk ${ }^{2}$ \\ ${ }^{1}$ Department of Epidemiology and Tropical Medicine in Gdynia, Military Institute of Medicine, Warsaw, Poland \\ ${ }^{2}$ Students' Scientific Circle of Travel Medicine, Medical University of Warsaw, Poland
}

\begin{abstract}
The aim of the article is to discuss issues associated with the occurrence of febrile illnesses in leisure and business travellers, with a particular emphasis on fevers of unknown origin (FUO). FUO, apart from diarrhoeas, respiratory tract infections and skin lesions, are one of the most common health problems in travellers to tropical and subtropical countries. FUO are manifestations of various diseases, typically of infectious or invasive aetiology. In one out of 3 cases, the cause of a fever in travellers returning from the hot climate zone is malaria, and therefore diagnostic tests should first aim at ruling out this specific disease entity. Other illnesses with persistent fever include dengue, enteric fever, viral hepatitis $A$, bacterial diarrhoeas and rickettsioses. Fever may also occur in travellers suffering from diseases of non-tropical origin, e.g. cosmopolitan respiratory tract or urinary tract infections, also, fever may coexist with other illnesses or injuries (skin rashes, bites, burns).
\end{abstract}

(Int Marit Health 2015; 66, 2: 77-83)

Key words: fever of unknown origin, travellers, tropics

\section{INTRODUCTION}

International travel has increased in recent years. The number of over one billion travellers recorded in 2012, described by the UNWTO as a historic milestone, continued to grow and rose by 5\% in 2013 [1]. According to Tourism Towards 2030 - A Global Overview created by the UNWTO, international tourist arrivals are predicted to reach 1.8 billion by 2030 [2]. Regrettably, travelling is an important factor in globalising infections and introducing pathogens into new regions [3]. That data should be taken into account while analysing travel-related morbidity. One of the most common health problems acquired by travellers going to tropical and subtropical destinations, beside diarrhoeas, respiratory tract infections and skin lesions, are fevers of unknown origin (FUO) [4].

FUO is classically defined by Petersdorf and Beeson as a prolonged febrile illness lasting at least 3 weeks, with repeated temperature measurements of $38.3^{\circ} \mathrm{C}$ or higher, defying diagnosis after 1 week of hospital inpatient evaluation. It is also described as fever that does not resolve spontaneously in the period expected for self-limited in- fection and whose cause cannot be ascertained despite considerable diagnostic effort [5]. There are various causes of unexplained fever. According to Cunha [6], infectious, rheumatic/inflammatory, neoplastic, or miscellaneous disorders can all be responsible for causing FUO.

\section{EPIDEMIOLOGY}

According to the GeoSentinel Surveillance Network and its database created between 1997 and 2006 based on 24,920 cases, $28 \%$ of returning travellers reported fever as a major reason for seeking medical care, similarly to $90 \%$ of travellers who were diagnosed with malaria, $82 \%$ with dengue, $87 \%$ with influenza, 96\% with leptospirosis, $87 \%$ with enteric fever, $100 \%$ with measles, and $72 \%$ with rickettsial infections [7]. In the study of Bottieu et al. [8], tropical infections were identified as a cause of fever in $36 \%$ of the cases, with malaria as a leading diagnosis, followed by rickettsial infections, dengue, acute schistosomiasis, enteric fever and invasive amoebiasis in lower numbers. Cosmopolitan infections were not rare as well - they accounted for $34 \%$ of all the cases, with respiratory tract infection, 
bacterial enteritis, mononucleosis-like syndrome, skin/soft tissue infection, and genitourinary infection as major causes. Among 613 French patients hospitalised due to a history of travel and fever, malaria was the most common diagnosis (75.2\%), while food-borne and water-borne infections, like gastroenteritis, typhoid fever or shigellosis were the second most common. Respiratory tract infections were the third most common cause of fever in those patients. In 13 cases dengue was reported as a cause of fever in the studied patients [9]. Among 774 German travellers, $27.9 \%$ reported fever, which was the second most frequent symptom after acute diarrhoea. In that group of patients, travellers with febrile/systemic diseases were mainly diagnosed for malaria, mononucleosis, and dengue. In 89 cases fever was accompanied by acute diarrhoea, caused mainly by Salmonella spp., Campylobacter spp. and Entamoeba spp. [10]. According to the study of Stienlauf et al. [11], febrile diseases accounted for $77 \%$ of all hospital admissions and were divided into 3 major causes: malaria, unidentified febrile disease and dengue. According to the analysis based on the data from the GeoSentinel Surveillance Network, $20 \%$ of travellers with fever remained undiagnosed [7]. According to Bottieu et al. [8], a definitive diagnosis was not established in $24 \%$ of the cases. In the study of Parola et al. [9], of 631 French travellers with fever, 50 remained with no confirmed diagnosis. As mentioned before, in the research performed by Stienlauf et al. [11], unidentified febrile diseases accounted for $21 \%$ of the cases. Data analysed by Bottieu et al. [8] showed that the highest rate of FUOs was among patients returning from North Africa and Middle East - almost $40 \%$ of fever cases from those regions remained undiagnosed.

In the GeoSentinel Surveillance Network survey [7], there are 10 regional classifications. A place of exposure turned out to be crucial in diagnosing returning travellers. Those who travelled to Sub-Saharan Africa and Oceania and reported fever as a chief symptom were most often diagnosed with malaria. Travellers coming back from South-East Asia and the Caribbean were more likely to be diagnosed with dengue. III travellers returning from South-Central Asia were most commonly diagnosed with enteric fever [12]. Chikungunya was more likely among patients returning from Indian Ocean Islands. Those diagnosed with rickettsial diseases were usually coming back from Southern Africa. Most of them suffered from tick-borne rickettsioses. Dermatologic conditions were more likely among travellers to Oceania, South-East Asia, South and Central America and the Caribbean [13]. According to the GeoSentinel study of travel-related infectious diseases reported in Europe, febrile systemic illnesses were usually spread from Sub-Saharan Africa, Indian Ocean Islands and Oceania. Plasmodium falciparum malaria was more frequently observed in patients coming back from Sub-Saharan Africa and Indian Ocean Islands while Plasmodium vivax malaria was more frequent in travellers from Oceania, Indian Ocean Islands and South America. Travellers returning from South-East Asia were commonly diagnosed with dengue, while those coming back from Sub-Saharan Africa were more likely to suffer from rickettsioses [13]. In another study rickettsioses were frequently recorded in travellers coming back from Sub-Saharan Africa as well [14]. Acute diarrhoea occurred mainly in travellers returning from North Africa, South-Central Asia and Middle East [13]. In the study of Bottieu et al. [8], Plasmodium falciparum, P. malariae, P. ovale, P. malariae, rickettsial infection, and acute schistosomiasis were reported mainly in patients who had returned from Africa, while Plasmodium vivax malaria, dengue, and enteric fever were the most frequent reasons for fever of tropical origin in travellers returning from Asia. In patients from Latin America fever was rarely caused by tropical infections - if so, almost only dengue and Plasmodium vivax malaria were reported as the reasons. According to another survey of Bottieu et al. [15], $P$. falciparum malaria, rickettsial infections, and acute schistosomiasis were almost exclusively diagnosed after a stay in Sub-Saharan Africa, while most cases of dengue and enteric fever were found in travellers returning from Southern Asia/Pacific. Dengue was the most frequent tropical cause of fever in travellers to Latin America.

\section{REASONS OF TRAVEL}

The survey by Wilson et al. [7] for the GeoSentinel Surveillance Network includes 5 reasons for travel - tourism, business, research/education, missionary/volunteer and visiting friends and relatives (VFR). Travellers VFRs are more likely to report fever after returning home than other groups of travellers, especially those who visited Sub-Saharan Africa, South-Central Asia, Indian Ocean Islands, Oceania and Latin America [12]. That type of travelling is a risk factor for travel-acquired illnesses in general and the VFR group of patients was significantly standing out from other types of travellers diagnosed with malaria and enteric fever [12]. Acute diarrhoea was more common among classic tourist travellers. P. falciparum malaria was also more frequent in VFRs, while $P$. vivax malaria was more likely to be reported in missionary or expatriate travellers. However, VFRs returning from Africa and Indian Ocean Islands were more frequently diagnosed with $P$. falciparum malaria compared with non-European travellers [13]. Tuberculosis and human immunodeficiency virus (HIV) infection as a reason of fever were much more often diagnosed in VFR travellers and foreign visitors or migrants [8]. 


\section{DEMOGRAPHIC FACTORS}

According to the GeoSentinel research conducted between 1997 and 2006, male travellers were more likely to be presenting with fever than females. $32 \%$ of ill returning travellers were men with fever and only $24 \%$ of that group were women presenting with this symptom [7]. Women are generally less prone to febrile illnesses and vector-borne diseases, such as malaria or rickettsioses [16]. There was no age difference between travellers presenting with fever and those without that symptom [7]. According to the analysis from the GeoSentinel Surveillance Network of illnesses in children after international travels, malaria was the most common cause of systemic febrile diseases, before viral syndromes (28\%), unspecified febrile illnesses (11\%), dengue and enteric fever (6\% each) [17]. In the study of Herbinger et al. [10], causes of fever were mainly malaria, mononucleosis and dengue. In children, however, the most frequent causes varied by age. In the group 0-4 years, acute diarrhoea was significantly more frequent than in other groups, while among children between 10 and 14 years, dengue was diagnosed more often. In adolescents aged between 15 and 19, the prevalence of mononucleosis as a cause of fever was significantly higher.

\section{PRE-TRAVEL MEDICAL CONSULTATION}

According to the study of Wilson et al. [7], 30\% of the travellers with fever didn't seek pre-travel consultation, while $27 \%$ claimed to have sought medical advice. In the study of Shlagenhauf et al. [3], pre-travel medical consultation was associated with noticeably lower morbidity for $P$. falciparum malaria. At the same time, patients who had had pre-travel consultation were less likely to get a severe illness. In the study of Bottieu et al. [8], only 33\% of Western travellers took malaria chemoprophylaxis before travelling and that percentage was even lower among expatriates (10\%) and VFR travellers (19\%). In another study, which seems important, a danger to be diagnosed with a vaccine-preventable cause of fever was doubled for VFRs as compared to other travellers, yet they sought pre-travel health consultation less frequently [7]. According to the data obtained between 1997 and 2007, women are more likely to seek for pre-travel heath information than men. However, there were more female travellers reporting adverse events related to medication, especially used as malaria prophylaxis [16]. In the survey by Wilson et al. [7] vaccine-preventable infections accounted for $3 \%$ of febrile illnesses in returning travellers. Typhoid fever, acute viral hepatitis A and influenza A were the most frequent causes of fever in that group. Those infections were more frequent among VFRs.

\section{FEVER IN TRAVELLERS - THE MOST FREQUENT ACCOMPANYING SYMPTOMS AND DIAGNOSES}

Prolonged fever is a significant symptom in travellers returning from the tropics. As there are many different factors affecting morbidity in returning travellers, fever as a symptom is difficult to attribute to any specific illness in the first place. The GeoSentinel Global Surveillance System is the widest source data on travel-related illnesses. It was established in 1995 to perform provider-based monitoring of travel-related morbidity among international travellers [18]. According to the study among 24,290 ill returning travellers between 1997 and 2006, 28\% of the patients reported fever as their main symptom. There were different symptoms accompanying fever, leading to different diagnoses. 35\% of ill travellers with fever had a febrile systemic illness, in 15\% of the cases it was a febrile diarrheal disease and $14 \%$ of the patients suffered from fever and respiratory disease. There were also cases connected with dermatologic and genitourinary diagnoses as well as non-diarrheal gastrointestinal diagnoses (4\% each). There was also a significant group of patients (22\%) in whom the diagnosis was defined as an unspecified febrile illness [7].

In systemic febrile illnesses, the most frequent diagnosis was malaria, which was found in $21 \%$ of all ill returned travellers with fever. The second most common reason was dengue (6\%) before enteric fever caused by Salmonella typhi or paratyphi infection and rickettsial diseases (2\% each). Mononucleosis syndromes accounted for $1.4 \%$ of all the travellers with fever. They were caused by e.g. Epstein-Barr virus (EBV) infection, cytomegalovirus (CMV) infection, acute HIV infection or toxoplasmosis [7]. According to Harvey et al. [18], systemic febrile diseases accounted for $14 \%$ of all ill returning travellers and in that group 59\% were accounted for by 5 diagnoses: Plasmodium falciparum malaria (19\%), viral syndrome without rash (17\%), uncomplicated dengue (11\%), unspecified febrile disease (<3 weeks) (8\%), and EBV infection (4\%). In the study of 17,353 cases conducted between 1996 and 2004, based on the GeoSentinel Database, systemic febrile diseases were most frequently found in returned travellers. Those conditions were most commonly caused by malaria, then dengue, mononucleosis due to EBV or CMV infection, rickettsial infection and Salmonella typhi or Salmonella paratyphi infection [4].

\section{MALARIA}

According to Wilson et al. [7], malaria was the most frequent diagnosis reported in $21 \%$ of returning travellers with fever. Malaria caused by Plasmodium falciparum accounted for $14 \%$ of febrile returned travellers and $66 \%$ of all the malaria patients in that study. It was the most frequent 
among travellers returning from Oceania, Pacific Islands and Sub-Saharan Africa. What is important, among 12 deaths reported in that survey, 4 were caused by $P$. falciparum malaria. According to Leder et al. [19], a total of 1,140 malaria cases were reported to the GeoSentinel between 1997 and 2002. 93\% of patients with $P$. falciparum infection, 95\% with $P$. vivax, $83 \%$ with $P$. ovale, and $95 \%$ with $P$. malariae reported fever while presenting to GeoSentinel units. Malaria hospitalised patients varied by type of the disease - $60 \%$ were diagnosed with $P$. falciparum, in cases of $P$. vivax, $P$. ovale and $P$. malariae the numbers were much lower (45\%, $30 \%$ and $13 \%$, respectively). Cerebral malaria reported in P. falciparum infection occurred in $2.4 \%$ of the cases. Types of malaria in patients with fever are closely connected with the visited region. P. falciparum infection was more frequently observed in patients from Sub-Saharan Africa while $P$. vivax malaria was more frequent in travellers from Oceania, Indian Ocean Islands and South America [13, 20]. Transmission of malaria outside endemic area is rare, although possible (airport and baggage malaria, blood transfusion and intravenous drugs use). In 2012, in the United States there were 1,687 malaria cases, of which 1,683 were imported [21]. On European continent France has the highest number of travellers returning from endemic zones with malaria [3]. Pregnant women and immunoincompetent patients are at higher risk of infection [22].

\section{DENGUE}

Wilson et al. [7] have established that dengue was diagnosed in $6 \%$ of returning travellers with fever. However, it is probable that, because of some reasons, e.g. a short incubation period as well as its possible mild and nonspecific symptoms, a lot of cases were omitted. In the study of Gautret et al. [13], dengue was the second most frequent cause of fever in travellers and it is considered one of the most common causes of fever in ill returned travellers. According to Shlagenhauf et al. [3], the proportionate morbidity of dengue among European travellers increased in the period between 2008 and 2012 from 22 to 36 cases. Dengue was the most frequent reason of systemic febrile illness in travellers coming back from South-East Asia [7]. According to the analyses by the GeoSentinel Surveillance System, conducted in the United States in the years 1997-2011, of the 1,802 returned travellers with fever, $11 \%$ were diagnosed with dengue [18]. More than 100 countries in Africa, Americas, South-East Asia and Eastern Mediterranean are endemic. Over the past 50 years there has been a 30 -fold increase in the incidence of dengue [23]. Studies show that $14-87 \%$ of all dengue infections are symptomatic [24]. That is why dengue should always be considered in patients returning from endemic regions.

\section{ENTERIC FEVER}

In the study of Wilson et al. [7], Salmonella enterica serovar typhi or paratyphi infection occurred in $2 \%$ of travellers with fever and was most frequently diagnosed in people returning from South-Central Asia. Although the most common cause of enteric fever worldwide is S. typhi, S. paratyphi may be a more common cause of this illness in travellers, which may be explained by a growing number of tourists vaccinated against S. typhi [25]. A study conducted on case records from a Swedish database of notifiable and communicable diseases [26] concludes that the highest risk of being infected with enteric fever is connected with travel to India and neighbouring countries (41.7/100,000 people for S. typhi infection and $37.5 / 100,000$ for S. paratyphi), followed by Middle East (5.91/100,000 and 3.64/100,000, respectively) and Africa (3.33/100,000 for both typhoid and paratyphoid fever). Other studies show that travel to Indian subcontinent might be associated with an even 18 times higher risk of contracting enteric fever than to any other region [25].

\section{RICKETTSIA}

According to a study of Jensenius et al. [14], in the period 1984-2004 more than 450 cases of rickettsioses were reported among travellers. According to the GeoSentinel network, from 1996 to 2008 there were 280 cases (231 spotted fever, 16 scrub typhus). Between 2007 and 2011,300 cases of rickettsioses were reported in travellers (267 spotted fever, 17 murine typhus and 14 scrub typhus) [19]. In a study performed by French physicians between 2004 and 2013, African tick-bite fever (ATBF) and Mediterranean spotted fever (MSF) turned out to be the two most frequent spotted fevers in travellers. According to that data, ATBF was the second most frequent diagnosis of fever in travellers returning from Sub-Saharan Africa. The rates of the disease were particularly high among tourists. MSF is endemic in the Mediterranean area and the cases were reported mainly in people travelling in July and August [27].

\section{MONONUCLEOSIS}

According to the GeoSentinel study from 2003 mononucleosis syndromes occurred in $1.4 \%$ of travellers returning with a systemic febrile illness [7]. Infectious mononucleosis (IM) is a disease mainly caused by EBV, but CMV, HIV or toxoplasmosis may also be responsible for causing mononucleosis-like diseases. In the study carried out by Bottieu et al. [8] over the period of 5 years, the four diseases accounted for $4 \%$ of all imported fevers, being the third most common diagnosis after bacterial enteritis and respiratory tract infections. The IM-like syndrome was most widespread in travellers coming back from Asia (7\%) before America (5\%), and Africa (2.5\%). Later on, the study showed that 
IM-like syndromes in travellers were mainly caused by CMV infection and less frequently by the classic EBV [8].

\section{GASTROINTESTINAL SYMPTOMS AND FEVER}

Fever in returning travellers is often accompanied by gastrointestinal symptoms, mainly diarrhoea. In the French survey, there were cases of fever accompanied by gastroenteritis, enteric fever, shigellosis, dysentery, non-typhoidal salmonellosis and amoebiasis [9].

\section{RESPIRATORY SYMPTOMS AND FEVER}

In patients presenting with respiratory illness and fever, the upper respiratory tract was affected more often $(58 \%$ of the patients). In $11 \%$ of febrile respiratory illnesses the reason was bacterial pneumonia, while $8 \%$ of those patients developed influenza or influenza-like illness. Other causes of fever connected with respiratory tract diseases were acute unspecified respiratory infection, bronchitis, tonsillitis and acute sinusitis [7]. In a survey performed by Australian researchers, respiratory infections were the second most common reason of fever in returning travellers. Infections of the upper respiratory tract were extremely common - and were the cause of $50 \%$ of all admissions. Another common cause of fever was bacterial pneumonia (25\% of the cases in the Australian research study), although it was most frequent in patients aged more than 40 years. Influenza was the second most common reason of fever among patients with respiratory infections; it affected $20 \%$ of patients in the Australian research [28]. Respiratory tract infections can be caused by endemic pathogens: in Europe and North America - Legionella pneumophila (ship cruising), in Latin America - Histoplasma, in Africa - Schistosoma and in South-East Asia - geohelminths. The most widespread cosmopolitan bacterial pathogens are Streptococcus pneumoniae, Haemophilus influenzae, group A Streptococci, Corynebacterium diphtheriae, Mycobacterium tuberculosis. The most common virus causing respiratory tract infections in travellers is the Influenza virus [29]. Respiratory symptoms may also occur in systemic febrile illnesses, mainly malaria, enteric fever and leptospirosis, which may result in some diagnostic problems [30].

\section{DERMATOLOGICAL SYMPTOMS AND FEVER}

There are very few studies analysing fever accompanied by skin manifestations. In his study Hochedez et al. [31], reported of 62 travellers presenting with fever and exanthema. In those cases, exanthema was macular in $39(63 \%)$ cases, maculopapular in $27 \%$, and maculopapular with eschar in $10 \%$ of the patients. Oral enanthema was observed in $13 \%$ of travellers with fever and exanthema. The aetiology was confirmed in $92 \%$ of the cases and there were 3 leading diagnoses - chikungunya (35\%), dengue
(26\%), and ATBF (10\%). In the study of Bottieu et al. [15], dermatological symptoms accompanying fever occurred in specific illnesses - enlarged lymph nodes and skin ulcers in rickettsial infections, and skin rash in dengue and rickettsial infection.

\section{COSMOPOLITAN INFECTIONS AND FEVER}

One should remember that there is a wide range of cosmopolitan infections causing fever in returning travellers such as common respiratory and urinary tract infections, HIV infection, CMV infection and toxoplasmosis or tuberculosis. Therefore, these illnesses should also be included in differential diagnosis [7].

\section{DIFFERENTIATING MALARIA FROM ENTERIC FEVER AND DENGUE}

Malaria, enteric fever and dengue being the most common causes of fever in returning travellers have overlapping and non-specific symptoms. Diagnostic problems caused by those illnesses are best illustrated by the fact that enteric fever and malaria were once merged (due to the absence of reliable differentiating methods) by Woodward into a clinical entity named typhomalaria [32]. One of the first to differentiate malaria and enteric fever was Osler. He described malaria as typically presenting with malarial paroxysm (starting with chills, then fever and finally sweats) and intermittent fever. Enteric fever was described as a disease typically characterised by relative bradycardia and a stepwise fever increasing over a period of 1 week [33]. Some uncertainty is always present regarding temporal changes of laboratory parameters. For example, it is known that leukopoenia occurs early in dengue but there is no consensus as to when thrombocytopoenia occurs [34]. The studies presented below try to find most specific clinical and laboratory findings to help differentiate between the two illnesses. According to Cooper et al. [34], rash is most common in dengue (usually widespread blanching exanthema) and if present in enteric fever it appears as rose spots but it does not occur in malaria. Organomegaly is most often found in malaria and can occur in enteric fever but it is not found in dengue. In dengue, laboratory findings include normal C-reactive protein (CRP) (although high CRP does not exclude the disease) and low white cell count. Normal CRP and leukopoenia and/or thrombocytopoenia were found in up to $1 / 3$ dengue cases but not in malaria or enteric fever patients. Thrombocytopoenia (platelets, PLT $<100 \times 10^{9} / \mathrm{L}$ ) was found in $1 / 3$ dengue cases and $1 / 2$ malaria cases. Malaria features a specific combination of laboratory patterns, which include thrombocytopoenia, leukopoenia and elevated: CRP, lactate dehydrogenase, and bilirubin. Finally, elevated alanine amino-transferase was found to be most specific for enteric fever. According to Bottieau et al. [15] 
malaria should be considered first. Symptoms found to be most specifically associated with malaria are splenomegaly, thrombocytopoenia, fever without localised symptoms and hyperbilirubinaemia. Only when malaria has been ruled out, should other possible aetiologies be considered. Skin rash and skin ulcer strongly suggest rickettsial infection which can be ruled out by leukocytosis. Skin rash (best predictor), thrombocytopoenia and leukopoenia are most commonly found in dengue. Eosinophilia is associated with acute schistosomiasis. Abdominal symptoms, enlarged spleen and elevated liver enzymes suggest diagnosis of enteric fever. Kutsuna et al. [35] proposed a diagnostic algorithm for malaria, enteric fever and dengue which combines most specific laboratory findings with the visited part of the world. Returning from Africa was strongly associated with malaria. Returning from elsewhere than Africa with CRP $<10 \mathrm{mg} / \mathrm{L}$ was characteristic for dengue. Patients returned from South Asia, with CRP $>10 \mathrm{mg} / \mathrm{L}$ and $\mathrm{PLT}>15$ cells $/ \mathrm{mm}^{3}$ were most likely to have enteric fever.

\section{CLINICAL PRESENTATION AFTER TRAVEL}

Fever caused by a tropical infection appears within 1 month after returning from or arrival in a tropical or subtropical region in $82 \%$ of the ill travellers, with all dengue and rickettsial infections included. However, malaria is the most common tropical infection that presented with fever within the second or even third month on return [8]. According to Wilson et al. [7] most infections in returning travellers have incubation periods shorter than 30 days. However, there are diseases, like malaria ( $P$. vivax infection), viral hepatitis or tuberculosis that can manifest within 30 (or more) days on travellers' return. The same study showed that the majority of patients who suffered from dengue sought medical assistance within 1 week on their return. $65 \%$ of patients with $P$. falciparum malaria and only $27 \%$ of patients with $P$. vivax consulted a physician within 2 weeks on their return. A great number of patients sought medical advice after a much longer period of time (within 16 weeks) including $45 \%$ of patients with $P$. vivax malaria and $34 \%$ travellers with viral hepatitis (mostly viral hepatitis A). In early-onset fever tropical infections were found in $41 \%$ of all cases. Malaria (in $92 \%$ caused by $P$. falciparum) was the most frequent cause of fever, followed by dengue, rickettsial infection, acute schistosomiasis, and enteric fever [15].

\section{APPROACH TO A PATIENT WITH FEVER OF UNKNOWN ORIGIN}

When collecting history, a physician should take special interest in the patient's recent travels. He/she should ask about the visited countries as well as about the visited regions, as some pathogens can only be present in certain parts of a given country, e.g. in rural areas. A physician should inquire about exposure history: sexually transmitted diseases, food-borne illnesses, vector-borne infections; fever may also coexist with other illnesses or injuries (skin rashes, bites, burns). A physician should also consider cosmopolitan pathogens and common infections such as urinary tract infections and upper respiratory tract infections, and never assume that fever may only be travel-related. A short incubation period of a disease (less than 21 days) indicates dengue, chikungunya, malaria, enteric fever, CMV, EBV mononucleosis, Q fever, viral haemorrhagic fever or a rickettsial infection, whereas a long incubation period (over 21 days) is most often associated with malaria, tuberculosis, viral hepatitis A, amoebic liver abscess and acute schistosomiasis. Most patients with non-focal fever would have cosmopolitan infection, malaria, enteric fever, dengue or rickettsial infection. When in doubt, malaria should be ruled out first as it is the deadliest of the illnesses percurrent with fever. What is more, in one out of 3 cases, the cause of a fever in travellers returning from tropical destinations is malaria, for that reason diagnostic procedures undertaken by medical professionals should primarily be oriented towards identifying or eliminating this specific disease entity $[30,36]$.

\section{REFERENCES}

1. World Tourism Organization. UNWTO Annual Report 2013, Madrid.

2. World Tourism Organization. UNWTO Tourism Towards 2030 - Global Overview, Republic of Korea.

3. Schlagenhauf $P$, Weld L, Goorhuis A et al. Travel-associated infection presenting in Europe (2008-2012): an analysis of EuroTravNet longitudinal, surveillance data, and evaluation of the effect of the pre-travel consultation. Lancet Inf Dis 2014; 15: 55-64.

4. Freedman DO, Weld LH, Kozarsky PE et al. GeoSentinel Surveillance Network. Spectrum of Disease and Relation to Place of Exposure among III Returned Travelers. N Engl J Med 2006; 354: 119-130.

5. Arnow PM, Flaherty JP. Fever of unknown origin. Lancet 1997; 350: $575-578$.

6. Cunha BA. Fever of unknown origin: focused diagnostic approach based on clinical clues from the history, physical examination and laboratory tests. Infect Dis Clin North Am 2007; 21: 1137-1188.

7. Wilson ME, Weld LH, Boggild A et al. GeoSentinel Surveillance Network. Fever in returned travelers: results from GeoSentinel surveillance network. Clin Infect Dis 2007; 44: 1560-1568.

8. Bottieu E, Clerinx J, Schrooten W et al. Etiology and outcome of fever after a stay in the tropics. Arch Intern Med 2006; 166: 1642-1648.

9. Parola P, Soula G, Gazin P, Foucault C, Delmont J, Brouqui P. Fever in travelers returning from tropical areas: prospective observational study of 613 cases hospitalised in Marseilles, France, 1999-2003. Travel Med Infect Dis 2006; 4: 61-70.

10. Herbinger KH, Drerup L, Alberer M, Nothdurft HD, von Sonnenburg F, Loscher T. Spectrum of imported infectious diseases among children and adolescents returning from the tropics and subtropics. J Trav Med 2012; 19: 150-157.

11. Stienlauf S, Segal G, Sidi Y, Schwartz E. Epidemiology of travel-related hospitalization. J Travel Med 2005; 12: 136-141.

12. Boggild AK, Geduld J, Libman M et al. Travel-acquired infections and illnesses in Canadians: surveillance report from CanTravNet surveillance data, 2009-2011. Open Med 2014; 8: e20-e32. 
13. Gautret P, Schlagenhauf P, Gaudart J et al.; GeoSentinel Surveillance Network. Multicenter Euro TravNet/GeoSentinel study of ravel-related infectious diseases in Europe. Emerg Inf Dis 2009; 15: 1783-1790.

14. Jensenius M, Davis X, von Sonnenburg F et al. GeoSentinel Surveillance Network. Multicenter GeoSentinel Analysis of rickettsial diseases in international travelers, 1996-2008. Emerg Inf Dis 2009; 15: 1791-1798.

15. Bottieau E, Clerinx J, Van den Enden E et al. Fever after a stay in the tropics: diagnostic predictors of the leading tropical conditions. Medicine (Baltimore) 2007; 86: 18-25.

16. Schlagenhauf $P$, Chen $\mathrm{LH}$, Wilson $\mathrm{ME}$ et al. Sex and gender differences in travel-associated disease. Clin Infect Dis 2010; 50: 826-832.

17. Hagmann S, Neugebauer R, Schwartz E et al. Illness in children after international travel: Analysis from the GeoSentinel Surveillance Network 2009. Pediatrics 2010; 125: e1072-e1080.

18. Harvey K, Esposito DH, Han P et al. Surveillance for travel-related disease - GeoSentinel Surveillance System, United States, 1997-2011. Morb Mortal Wkly Rep Surveill Summ 2013; 62:1-23.

19. Leder K, Torresi J, Libman MD et al. GeoSentinel Surveillance Network. GeoSentinel surveillance of illness in returned travelers, 2007-2011. Ann Intern Med 2013; 158: 456-468.

20. Norman F, Monge-Maillo B, Martínez-Pérez A, Perez-Molina JA, López-Vélez R. Parasitic infections in travelers and immigrants: part I protozoa. Future Microbiol 2015; 10: 69-86.

21. Cullen KA, Arguin PM. Centers for Disease Control and Prevention. Malaria surveillance - United States, 2012. MMWR Surveill Summ 2014; 63: 1-22.

22. Askling $\mathrm{HH}$, Brunee $\mathrm{F}$, Burchard $\mathrm{G}$ et al. Management of imported malaria in Europe. Malaria J 2012; 11: 328.

23. World Health Organization. Dengue and severe dengue. Fact sheets No. 117/2015. Available at: http://www.who.int/mediacentre/ factsheets/fs117/en.

24. Jelinek T. Dengue fever in international travelers. Clin Infect Dis 2000; 31: 144-147.
25. Connor BA, Schwartz E. Typhoid and paratyphoid fever in travellers. Lancet Infect Dis 2005; 5: 623-628.

26. Ekdahl K, de Jong B, Andersson Y. Risk of travel-associated typhoid and paratyphoid fevers in various regions. J Travel Med 2005; 12:197-204.

27. Delord M, Socolovschi C, Parola P. Rickettsioses and Q fever in travelers. Travel Med Infect Dis 2014; 12: 443-458.

28. O'Brien D, Tobin S, Brown GV, Torresi J. Fever in returned travelers: review of hospital admissions for a 3-year period. Clin Infect Dis 2001; 33: 603-609.

29. Korzeniewski K, Nitsch-Osuch A, Lass A, Guzek A. Respiratory infections in travelers returning from the tropics. Adv Exp Med Biol 2015; 849: 75-82.

30. Johnston V, Stockley JM, Dockrell D et al. The British Infection Society and the Hospital for Tropical Diseases. Fever in returned travellers presenting in the United Kingdom: recommendations for investigation and initial management. J Infect 2009; 59: 1-18.

31. Hochedez P, Canestri A, Guihot A, Brichler S, Bricaire F, Caumes E. Management of Travelers with Fever and Exanthema, Notably Dengue and Chikungunya Infections. Am J Trop Med Hyg 2008; 78: 710-713.

32. Bynum B. Discarded diagnoses - Typhomalaria. Lancet 2002; 360: 1339.

33. Cunha BA, Gran A, Munoz-Gomez S. Typhoid fever vs. malaria in a febrile returning traveler: typhomalaria revisited - an Oslerian perspective. Travel Med Infect Dis 2013; 11: 66-69.

34. Cooper EC, Ratnam I, Mohebbi M, Leder K. Laboratory features of common causes of fever in returned travelers. J Travel Med 2014; 21: 235-239.

35. Kutsuna S, Hayakawa K, Kato Y et al. Comparison of clinical characteristics and laboratory findings of malaria, dengue, and enteric fever in returning travelers: 8-year experience at a referral center in Tokyo, Japan. J Infect Chemother 2015; 21: 272-276.

36. Korzeniewski K. Travel medicine. Department of Epidemiology and Tropical Medicine, Military Institute of Medicine. Warsaw 2014; pp. 292-295 